\title{
Eficiência reprodutiva e enfermidades reprodutivas em rebanho Nelore PO na região oeste do Estado do Pará, Brasil
}

\author{
[Reproductive efficiency and reproductive diseases of a Nelore cattle herd raised in the west region \\ of Pará State, Brazil]
}

\section{"Artigo Científico/Scientific Article"}

\author{
Antonio Humberto Hamad Minervino ${ }^{1 *}$, Haroldo Francisco Lobato Ribeiro ${ }^{2}$, \\ Kedson Alessandri Lobo Neves ${ }^{1}$, Adriana Caroprezo Morini ${ }^{1}$, Raimundo Alves Barrêto Júnior ${ }^{3}$, \\ Carolina Akiko Sato Cabral Araújo ${ }^{4}$, William Gomes Vale ${ }^{1}$
}

\author{
${ }^{1}$ Universidade Federal do Oeste do Pará (UFOPA), Santarém-PA, Brasil. \\ ${ }^{2}$ Instituto de Produção e Saúde Animal, Universidade Federal Rural da Amazônia (UFRA), Belém-PA, Brasil. \\ ${ }^{3}$ Universidade Federal Rural do Semi-Árido (UFERSA), Mossoró-RN, Brasil. \\ ${ }^{4}$ Universidade Federal Rural de Pernambuco (UFRPE), Recife-.PE, Brasil. \\ *Autor para correspondência/Corresponding author: E-mail: ah.minervino@gmail.com
}

\begin{abstract}
Resumo
O presente estudo objetivou avaliar a eficiência reprodutiva e identificar a incidência de enfermidades reprodutivas em animais PO da raça Nelore através de exame clínico reprodutivo das fêmeas e levantamento retrospectivo, através de registros da propriedade, obtendo o período de serviço (PS) e intervalo entre partos (IEP). O PS e o IEP médios foram de 224 e 510 dias respectivamente. Os índices reprodutivos de IEP e PS encontrados no presente estudo, foram maiores aos relatados na literatura para animais da raça Nelore, criados no ambiente amazônico. A taxa de prenhez foi de 70,7\%. As principais ocorrências reprodutivas foram o anestro prolongado $(36,5 \%)$ e o anestro pós-parto $(31,7 \%)$. Os índices reprodutivos dos animais durante a estação chuvosa foram mais eficientes em relação à estação seca, provavelmente devido à melhor qualidade das pastagens. A identificação precoce dos distúrbios reprodutivos permite o tratamento ou descarte dos animais, reduzindo custos e melhorando os índices reprodutivos dos rebanhos.
\end{abstract}

Palavras-chave: bovinos; infertilidade; período de serviço; intervalo entre partos.

\begin{abstract}
The present study aimed to evaluate the reproductive efficiency and establish the incidence of reproductive diseases in Nelore (pure breed) animals via rectal palpation in cows and a retrospective survey, through property records, obtaining days open (DO) and calving interval (CI). The DO and CI observed were 224 and 510 days respectively. The reproductive indexes of DO and CI found in the present study were higher than those reported in the literature for Nelore animals raised in the Amazonian environment. The pregnancy rate was $70.7 \%$. The most important diseases observed were pathological anestrous (36.5\%) and post-calving anestrous $(31.7 \%)$. The reproductive rates were more efficient in the rainy season, probably because of the better quality of pastures. The earlier identification and treatment of reproductive diseases is important to elevate the reproductive efficiency in cattle.
\end{abstract}

Keywords: bovine; infertility; calving interval; service period.

\section{Introdução}

O Estado do Pará apresenta grande importância na atividade pecuária da Amazônia, pois possui o quinto maior rebanho bovino brasileiro, com mais de 20 milhões de cabeças (IBGE, 2016), possuindo potencial para o incremento da atividade pecuária, devido a sua

vasta extensão territorial, e as suas condições climáticas peculiares, como alta insolação o ano inteiro, ausência de estação fria, alta temperatura e pluviosidade. Tais características climáticas propiciam um excelente desempenho das gramíneas tropicais (Minervino et al., 2008). 
Animais considerados puros ou de alta linhagem, ou seja, de genética superior, na maioria dos casos apresentam uma melhor eficiência tanto produtiva quanto reprodutiva, devido ao trabalho de melhoramento genético por meio dos testes de progênie, selecionando os mais resistentes e adaptados ao ambiente Além dos aspectos genéticos, de outros fatores importantes como a adaptação ao ambiente, a sanidade e a nutrição dos rebanhos, incluindo a suplementação mineral (Aragón et al., 2001) para que os animais possam expressar todo o seu potencial genético. Bem como o manejo adotado, apresentam efeitos diretos sobre a eficiência reprodutiva (Bustamante et al., 1997; Fonseca et al., 2002). Os índices reprodutivos como o Período de Serviço (PS), que é o número de dias entre o parto e a concepção, e o intervalo entre partos (IEP), são importantes parâmetros na avaliação da eficiência reprodutiva dos rebanhos e quanto menor esses períodos, melhor a capacidade reprodutiva.

Por outro lado, os distúrbios reprodutivos são responsáveis, em parte, pela baixa eficiência reprodutiva dos rebanhos, e especialmente em animais PO, devendo por isso, serem controlados de maneira mais eficiente (Sheldon et al., 2006), pois, alguns desses problemas apresentam caráter hereditário, devendo por isso ser precocemente identificados e, uma vez identificados, os animais acometidos devem ser sumariamente descartados, garantindo que sua hereditariedade patológica, não seja transmitida através dos genes deletérios transmissores desses distúrbios, não interfiram de forma depreciativa no melhoramento genético dos rebanhos (Grunert et al., 2005).

Vale (1979) estudando a incidência de vacas Nelore repetidoras de estro em dois diferentes rebanhos, na região oriental do Estado do Pará, controlados durante o período de dois anos, encontrou para o primeiro rebanho uma prevalência de $21 \%$ e $6,6 \%$ de distúrbios reprodutivos, enquanto para o segundo rebanho $18 \%$ e $6 \%$, no primeiro e segundo ano, respectivamente. As principais causas responsáveis pela repetição de estros nas fêmeas examinadas foram cistos foliculares e cistos luteinizados, mucometra, endometrites, piometra, salpingite e hidrossalpingite, aderências tubo-ovarianas e tumores ovarianos. O mesmo autor preconizou a necessidade do descarte sumário de animais portadores de patologias de fundo hereditário.

Examinando 9.517 tratos genitais de fêmeas azebuadas abatidas em dois diferentes matadouros da cidade de Belém, Pará, Vale et al. (1984) observaram que $4.279 \quad(44,97 \%)$ fêmeas encontravam-se gestantes e, que entre essas, 505 $(5,31 \%)$ apresentavam algum tipo de distúrbio da gestação a termo. Ademais, das 5.238 fêmeas não gestantes $(55,03 \%), 1.644(17,27 \%)$ apresentavam algum tipo de anomalia nos ovários ou outros segmentos do trato genital, levando estes animais a alguma forma de infertilidade ou mesmo a esterilidade.

Segundo Gordon (1996), a reprodução é o fenômeno mais importante quando se leva em consideração a produção de bovinos, quer seja a produção de leite ou de carne, tendo-se em conta que uma vaca somente será produtiva, se no período de um ano, produzir um bezerro e ficar novamente gestante.

No Pará, existem poucos estudos que identifiquem os distúrbios reprodutivos que ocorrem com maior frequência em bovinos, especialmente em animais PO. Assim sendo, o presente estudo visou estabelecer os índices reprodutivos e quantificar as principais enfermidades passíveis de identificação em rebanho de animais melhorados da raça Nelore, criados no oeste do Estado do Pará, bem como avaliar quais os possíveis fatores que interferem na eficiência reprodutiva.

\section{Material e Métodos \\ Local do estudo}

O presente estudo foi realizado em uma fazenda comercial com rebanho Nelore formado por cerca 1.500 animais, puro de origem (PO), localizada no município de Itaituba $\left(4^{\circ} 13^{\prime} 0^{\prime \prime} \mathrm{S}\right.$; $56^{\circ} 1^{\prime} 0 " \mathrm{~W}$ ), Estado do Pará. O clima da região caracteriza-se como tipo Am Amazônico de acordo com a classificação de Kõppen, com clima quente e úmido, e temperaturas médias, máximas e mínimas anuais oscilando respectivamente, entre $25^{\circ} \mathrm{Ce} 26^{\circ} \mathrm{C}, 30^{\circ} \mathrm{C}$ e $31^{\circ} \mathrm{Ce} 21^{\circ} \mathrm{C}$ e $23^{\circ} \mathrm{C}$, enquanto que a precipitação pluviométrica apresenta valores anuais oscilantes em torno de $2.000 \mathrm{~mm}$, com maior concentração de chuvas no período de janeiro a junho concentrando mais de $70 \%$ da precipitação anual, e o período menos chuvoso, julho a início de dezembro (Oliveira Júnior e Correa, 2001).

Este tipo de propriedade rural pode ser considerado como um modelo para a região, muito embora tenha como objetivo a produção de animais PO, tourinhos e novilhas para venda no mercado regional. 


\section{Animais, alimentação e manejo}

O manejo adotado na propriedade é do tipo extensivo, onde os animais ocupavam áreas de pastagens cultivadas compostas principalmente pelas espécies de gramíneas Brachiaria brizantha, Brachiaria humidicola e Panicum maximum, manejados em um sistema de rotação de pastagens, caracterizado por um número reduzido de piquetes com um longo período de ocupação, recebendo ainda suplementação mineral em cocho coberto $\mathrm{ad}$ libtum.

Ademais, o manejo reprodutivo rotineiro utiliza um exame ginecológico após 45 dias do período pós-parto (PPP), com utilização da inseminação artificial (IA), através da observação de estro com sessões de observação de uma hora pela manhã e uma hora à tarde, com apoio de um rufião com buçal marcador. As vacas que não emprenham ao primeiro serviço são reinseminadas e após são repassadas com touros de comprovada fertilidade. O diagnóstico de gestação é feito através de palpação retal, 45 dias após a primeira ou segunda IA e aos 60 dias após a fêmea ser alocada com o touro. Nos casos de insucesso da fecundação, as vacas foram descartadas e enviadas para o abate. O manejo sanitário do rebanho foi realizado através de medidas profiláticas e de vacinação contra as principais enfermidades infecto-contagiosas que ocorrem na região, tais como Febre Aftosa, Raiva, Brucelose, assim como a vermifugação de todo o rebanho duas vezes por ano.

\section{Estudo retrospectivo}

Através de fichas de identificação e de avaliação reprodutiva dos registros da propriedade, entre os anos de 1999 até 2002, foi possível a tabulação dos dados e o cálculo dos índices reprodutivos. O intervalo entre partos (IEP) foi obtido pela subtração de duas datas de partos consecutivos do mesmo animal e o PS foi calculado subtraindo-se o período de gestação do IEP (Grunert et al., 2005).

Com base nas informações presentes nas fichas individuais das fêmeas, os animais foram divididos em dois grupos de acordo com o a estação do ano (seca ou chuvosa) em que ocorreu o período de 90 dias pós-parto de cada animal. Deste modo as fêmeas que tiveram o PPP entre os meses de dezembro a junho foram classificadas na estação chuvosa e as que apresentaram PPP de julho a novembro foram classificadas na estação seca. Os animais que por ventura pariram próximos ao fim das estações, tendo o PPP (90 dias) ocorrendo parte na estação seca, parte na chuvosa, foram desconsiderados para esta análise.

\section{Estudo prospectivo}

Através de exame clínico com ênfase ao trato reprodutivo, exames ginecológicos seqüenciados foram realizados com o objetivo de determinar o estado reprodutivo das mesmas. O exame ginecológico foi realizado através de exame retal onde foram examinados os diferentes segmentos do trato reprodutivo, tais como ovários, ovidutos, útero, cérvix, vagina e vulva. Havendo necessidade complementar foi realizada a vaginoscopia, seguindo a metodologia preconizada por Grunert et al. (2005). O exame ginecológico foi realizado em todas fêmeas em idade reprodutiva da propriedade, estabelecendo-se assim a ocorrência de diferentes distúrbios reprodutivos existentes no rebanho.

Ainda durante o exame ginecológico, foi avaliado se a fêmea encontrava-se prenhe ou não. A taxa de prenhez (TP) do rebanho foi estabelecida utilizando à seguinte formula:

$T P(\%)=($ PRENHEZ $/$ EXPOSTAS $) \times 100$

onde: PRENHEZ = número de fêmeas prenhes; EXPOSTAS = número de fêmeas expostas a touro ou inseminadas.

Durante o exame ginecológico os animais foram classificados em três categorias, de acordo com o estágio reprodutivo: vacas paridas $(\mathrm{n}=236)$ fêmeas plurípara com bezerro ao pé; vacas solteiras $(\mathrm{n}=123)$ - fêmeas plurípara em que o ultimo bezerro já havia sido desmamado; novilhas $(\mathrm{n}=154)$ - fêmeas primíparas.

\section{Análise estatística}

A análise estatística foi realizada utilizandose o software Minitab 14 (Minitab, 2000). Inicialmente foi feita uma estatística descritiva, com a obtenção de médias e desvios padrões de cada variável, com a divisão entre as categorias. Seguiu-se a aplicação do teste de Kruskal-Wallis para a comparação das variáveis reprodutivas entre as duas estações do ano. Os dados de prenhez positiva ou negativa foram convertidos em números e aplicou-se o teste de comparação de médias de Mood para avaliar se houve diferença da TP entre as três categorias animais.

\section{Resultados e Discussão}

As vacas paridas, solteiras e novilhas apresentaram taxa de prenhez de $87,3 \%, 62,6 \%$ e 
$51,9 \%$, respectivamente (Tabela 1). Houve diferença significativa da taxa de prenhez entre a categoria de vacas paridas, quando comparada a vacas solteiras e novilhas, no entanto, não houve diferença entre as categorias de vacas solteiras e novilhas. A média geral da taxa de prenhez, excluindo-se a divisão entre categorias, foi de $70,8 \%$, superior aos reportados para outros rebanhos da raça Nelore (Bustamante et al., 1997; Noronha et al., 1999).

Tabela 1. Taxa de prenhez nas diferentes categorias de fêmeas da raça Nelore, criadas na região Oeste do Pará.

\begin{tabular}{lccc}
\hline $\begin{array}{l}\text { Categoria animal } \\
\text { Animal type }\end{array}$ & $\begin{array}{c}\text { N animais prenhes } \\
\text { N pregnant }\end{array}$ & $\begin{array}{c}\text { N total } \\
\text { N total }\end{array}$ & $\begin{array}{c}\text { Taxa de prenhez (\%) } \\
\text { Pregnancy rate }\end{array}$ \\
\hline $\begin{array}{l}\text { Vacas paridas } \\
\begin{array}{l}\text { Cows in lactation } \\
\text { Vacas solteiras }\end{array}\end{array}$ & 206 & 236 & $87,3^{\mathrm{a}}$ \\
$\begin{array}{l}\text { Open cows } \\
\text { Novilhas }\end{array}$ & 77 & 123 & $62,6^{\mathrm{b}}$ \\
Heifers & & & $51,9^{\mathrm{b}}$ \\
\hline Total & 80 & 154 & 70,8 \\
\hline $\begin{array}{l}\text { N = Número. Letras diferentes na mesma coluna apresentam diferença significativa pelo teste de média de Mood }(\mathrm{p}> \\
\text { 0,05). Same symbol in the same column present significative difference by Mood's median test }(p>0,05) .\end{array}$
\end{tabular}

O período de serviço médio foi de 224,2 dias ( $\pm 122,8$ dias), enquanto que o IEP médio foi de 509,6 dias ( \pm 123 dias). Estes índices reprodutivos foram obtidos utilizando-se 265 informações de datas de partos e de inseminações. O elevado desvio padrão ocorreu provavelmente devido a presença de animais com problemas reprodutivos que faziam parte do rebanho, que foram descartados após o diagnóstico. Assim, possivelmente a presença dessas fêmeas, provocou uma elevação da média geral tanto do PS quanto do IEP, diminuindo a eficiência reprodutiva do rebanho.

Em outros estudos com animais da raça Nelore o PS encontrado foi muito inferior ao encontrados nos animais PO deste estudo, (Bustamante et al., 1997; Noronha et al., 1999; Cavalcante et al. 2001). Por outro lado, em um estudo com animais da raça Nelore criados no Estado do Maranhão, Cavalcante et al. (2001) obteve IEP um pouco inferior (431,8 dias) quando comparado ao observado no presente estudo.

A Tabela 2 apresenta a análise estatística entre os índices reprodutivos (PS e IEP) e a estação do ano.

Observa-se que houve uma diferença significativa entre as estações, com melhores resultados durante o período chuvoso do ano. Este resultado deve ter ocorrido em função da maior disponibilidade de forragem nesta estação, o que demonstra que o fator nutricional afeta a eficiência reprodutiva do rebanho. Provavelmente o regime de pasto não atenda as exigências nutricionais dos bovinos durante o período seco do ano, onde a escassez de chuvas promove uma queda quantitativa e qualitativa da produção das forrageiras tropicais, em especial no que diz respeito aos teores de proteína bruta e de minerais (Minervino et al., 2008), o que pode ter contribuído para a menor eficiência reprodutiva.

Tabela 2. Período de serviço (PS) e do intervalo entre partos (IEP) em rebanho da raça Nelore na região do Oeste do Estado do Pará, em relação a estação do ano.

\begin{tabular}{lcc}
\hline $\begin{array}{l}\text { Variável } \\
\text { Variable }\end{array}$ & $\begin{array}{c}\text { PS } \\
\text { Days open }\end{array}$ & $\begin{array}{c}\text { IEP } \\
\text { Calving interval }\end{array}$ \\
\cline { 2 - 3 } & $\begin{array}{c}\text { Média } \pm \text { desvio padrão } \\
\text { Mean } \pm \text { standard deviation }\end{array}$ & $\begin{array}{c}\text { Média } \pm \text { desvio padrão } \\
\text { Mean } \pm \text { standard deviation }\end{array}$ \\
\hline $\begin{array}{l}\text { Chuvosa }(\mathrm{n}=141) \\
\text { Rainy }\end{array}$ & $153,3 \pm 106,2^{\mathrm{a}}$ & $438,3 \pm 106,2^{\mathrm{a}}$ \\
Seca $(\mathrm{n}=124)$ & $304,8 \pm 85,1^{\mathrm{b}}$ & $590,8 \pm 84,1^{\mathrm{b}}$ \\
Dry & & \\
\hline $\mathrm{N}=$ número de animais. Letras diferentes na mesma coluna apresentam diferença significativa $(\mathrm{P}<0,01)$. \\
Different symbol in the same column present significative difference $(P<0,01)$.
\end{tabular}

Nutrição inadequada é uma reconhecida causa de redução de fertilidade de gado sob pastejo de gramíneas tropicais. Uma dieta de restrição de energia afeta a função ovariana em novilhas e vacas, reduzindo o diâmetro do folículo dominante e o desenvolvimento dos demais folículos (Rhodes 
et al., 1996; Wiltbank et al., 2002). Em razão desses fatores nutricionais como energia (IMS e gordura) e proteína presentes na dieta influenciarem positiva ou negativamente a reprodução bovina, estratégias de suplementação dietética podem ser adotadas de maneira efetiva para obter resultados favoráveis à fertilidade da fêmea bovina (Sartori e Guardieiro, 2010). Deste modo, além do melhoramento genético, o manejo nutricional e sanitário adequado são essenciais para a obtenção de índices produtivos e reprodutivos satisfatórios (Fonseca et al., 2002).

Em um estudo realizado na região Amazônia, Cavalcante et al., (2001) reportaram um PS de 146 dias, bem inferior á média geral obtida neste trabalho (224,2 dias), entretanto, ao compararmos estes resultados com a média do PS durante a estação chuvosa, percebe-se que são muito próximos. O provável déficit nutricional ocorrido durante o período seco do ano pode ter sido o responsável pelo aumento de quase $100 \%$ nos valores do PS (Tabela 2), o que elevou a média geral a valores insatisfatórios. É válido lembrar que a média do PS no período chuvoso, bem como a média obtida por Cavalcante et al. (2001), encontram-se acima dos valores considerados adequados para bovinos de corte, onde o PS não deve ultrapassar os 100 dias (Grunert et al., 2005), para obtenção de um bezerro vaca/ano.A média do IEP foi ligeiramente inferior à obtida em rebanho Nelore no Mato Grosso do Sul, que foi de 514 dias, sendo superior à média em estudo na região Amazônia (Cavalcante et al. 2000). Contudo as diferenças no IEP, afora o fator genético, são acarretadas principalmente das particularidades do manejo, seja ele nutricional, sanitário ou reprodutivo, utilizado em cada uma das propriedades, sendo difícil realizar uma comparação entre os dados. Contudo, é possível se afirmar, que apesar de serem animais PO, era de se esperar uma melhor produtividade. O IEP está acima do considerado ideal e economicamente viável para gado de corte, que foi estabelecido em 380 dias aproximadamente (Grunert et al., 2005).

$\mathrm{Na}$ avaliação das enfermidades reprodutivas, observa-se que as vacas paridas apresentaram um baixo índice de distúrbios reprodutivos quando comparados às vacas solteiras no momento da realização dos exames ginecológicos. Apesar disso, o índice geral de animais com algum tipo de distúrbio reprodutivo foi relativamente elevado, chegando a $12,3 \%$ de todos os animais submetidos à avaliação clínica (Tabela 3). Enfermidades reprodutivas são responsáveis pela baixa eficiência dos rebanhos, com elevação do período de serviço e do intervalo de partos (Leite et al., 2001), podendo-se desta forma concluir que 150 animais que não estavam prenhes, $42 \%$ apresentavam algum tipo de alteração, sendo que $28,6 \%$ eram enfermidades reprodutivas enquanto que o restante $(13,3 \%)$ apresentava anestro prolongado pós-parto, provavelmente por deficiência nutricional.

Tabela 3. Apresentação geral dos distúrbios reprodutivos encontrados em rebanho da raça Nelore criados no Oeste do Estado do Pará.

\begin{tabular}{lccc}
\hline $\begin{array}{l}\text { Categoria } \\
\text { Type }\end{array}$ & $\begin{array}{c}\text { Animais examinados } \\
\text { Animals examined }\end{array}$ & \multicolumn{2}{c}{$\begin{array}{c}\text { Distúrbios reprodutivos no rebanho } \\
\text { Reproductive disorders }\end{array}$} \\
\cline { 3 - 4 } & & $\mathbf{N}$ & \% \\
\hline $\begin{array}{l}\text { Vacas paridas } \\
\text { Cows in lactation }\end{array}$ & 236 & 26 & 11,0 \\
$\begin{array}{l}\text { Vacas solteiras } \\
\text { Dry cows }\end{array}$ & 123 & 31 & 25,2 \\
Novilhas & & & 3,9 \\
Heifers & 154 & 6 & 12,3 \\
\hline Total & 513 & 63 & \\
\hline
\end{tabular}

$\mathrm{O}$ anestro prolongado foi considerado como a ausência de atividade reprodutiva em vacas solteiras, ou seja, que já desmamaram seus bezerros e consequentemente estão com um período de serviço maior que sete meses, enquanto que o anestro pós-parto foi considerado a ausência de atividade reprodutiva em vacas paridas, em que os bezerros já apresentam idade avançada.
Como a principal ocorrência reprodutiva observada foi o anestro prolongado (36,5\%), recomendou-se o descarte sumário destes animais do rebanho, com o objetivo de promover uma efetiva pressão de seleção, buscando acelerar o melhoramento genético. Da mesma forma, o anestro pós-parto também acometeu um elevado número de animais, entretanto é possível que outros fatores, provavelmente os de origem 
nutricional, possam ter interferido no retorno da atividade ovariana no PPP (Tabela 4). Assim, a recomendação para estes casos foi a da aplicação de técnicas de manejo, como o desmame temporário (método Chang), para estimular o retorno da atividade ovariana, associado ao tratamento com Prostaglandina $\left(\mathrm{PGF}_{2 \alpha}\right)$ em uma única aplicação de $2 \mathrm{~mL}$ por animal (Sincrocio ${ }^{\circledR}-$ Ouro Fino Saúde Animal) baseado em estudos que recomendam uma única aplicação de $\mathrm{PGF}_{2 \alpha}$ como tratamento do anestro pós-parto prolongado, visando reduzir o PS (Rao et al., 1991; Grunert et al., 2005).

$\mathrm{Na}$ Tabela 4 foram avaliados 63 animais que apresentaram distúrbios reprodutivos, e quantificados quanto a sua patologia e a frequência pode-se observar que as enfermidades diagnosticadas no rebanho estudado, são similares e de ocorrência semelhante aos reportados por outros autores, frequentes tanto em rebanhos de corte como de leite (Vale et al., 1979; Vale et al., 1984).

Tabela 4. Apresentação da freqüência de distúrbios reprodutivos em fêmeas da raça Nelore, criadas no oeste do Estado do Pará.

\begin{tabular}{|c|c|c|}
\hline \multirow[t]{2}{*}{$\begin{array}{l}\text { Distúrbio reprodutivo } \\
\text { Reproductive disease }\end{array}$} & \multicolumn{2}{|c|}{$\begin{array}{c}\text { Frequência } \\
\text { Frequency }\end{array}$} \\
\hline & $\mathbf{N}$ & $\%$ \\
\hline \multicolumn{3}{|l|}{ Alterações ovarianas } \\
\hline \multicolumn{3}{|l|}{ Ovarian disorders } \\
\hline Aderência ovariana & & \\
\hline Ovarian adherence & 1 & 1,6 \\
\hline Hipoplasia Ovariana & 1 & \\
\hline Ovarian hypoplasia & 1 & 1,6 \\
\hline \multicolumn{3}{|l|}{ Cistos ovarianos } \\
\hline \multicolumn{3}{|l|}{ Ovarian cysts } \\
\hline Cisto luteinizado & & \\
\hline Luteinized cysts & 3 & 4,8 \\
\hline Cisto folicular & & \\
\hline Follicular cysts & 2 & 3,2 \\
\hline \multicolumn{3}{|l|}{ Tumor de células da granulosa } \\
\hline Granulosa cell tumor & 1 & 1,0 \\
\hline Infecção uterina & & \\
\hline Uterine infection & 5 & 7,9 \\
\hline \multicolumn{3}{|l|}{ Alterações uterinas } \\
\hline \multirow{2}{*}{\multicolumn{3}{|c|}{ Uterine disorders }} \\
\hline Cérvix torta & & \\
\hline Sinuous cervix & 2 & 3,2 \\
\hline Útero infantil & 1 & \\
\hline Underdeveloped uterus & 1 & 1,6 \\
\hline Útero flácido & 1 & 16 \\
\hline Flaccid uterus & 1 & 1,0 \\
\hline Útero espesso & 1 & 16 \\
\hline Thick uterus & 1 & 1,0 \\
\hline \multicolumn{2}{|l|}{ Abortamento } & 32 \\
\hline Abortion & 2 & 3,2 \\
\hline Anestro prolongado & 23 & 36,5 \\
\hline Prolonged anestrous & 23 & \\
\hline Anestro pós-parto & 20 & 317 \\
\hline Pos-partum anestrous & 20 & 31,1 \\
\hline Total & 63 & 100 \\
\hline
\end{tabular}

Estudo realizado em animais prenhes (Leite et al., 2001) encontrou um índice de 9,15\% de abortamentos, portanto, superior ao observado neste estudo. As infecções uterinas têm causas e severidades variadas, em especial quanto a patogenicidade do agente etiológico, estado imunológico da vaca, condição corporal ruim ao parto, partos distócicos e falta de higiene em momentos de coberturas ou inseminações (Palhano, 2008). No presente estudo, os distúrbios reprodutivos relacionados ao útero apresentaram a terceira maior prevalência, com 12 animais acometidos e 19,1\% (12/63) de ocorrência. Desse modo, para controle dessas alterações uterinas, faz - se necessário, melhorar a higiene em momentos de inseminações, rigoroso controle sanitário do 
rebanho, propiciar boas condições de escore corporal as vacas ao parto, e exames ginecológicos periódicos (Nebel, 2003; Palhano, 2008). É importante ressaltar que no presente estudo, por se tratar de gado Nelore, a ocorrência de enfermidades reprodutivas é diferente de bovinos de outras raças, quer sejam de corte ou de leite (Vale et al., 1979; Vale et al., 1984).

Outras publicações demonstram que a ocorrência de distúrbios reprodutivos em bovinos, provoca uma elevação de 100 dias na duração do PS, o que reduz substancialmente a eficiência reprodutiva dos rebanhos (Grunert e Bertchold, 1999; Heather e Erb, 2000).

Os índices reprodutivos encontrados no presente estudo, relativo ao PS e IEP, foram superiores aos relatados na literatura para animais da raça Nelore, criados no ambiente amazônico. As principais enfermidades reprodutivas encontradas no presente estudo foram, $\mathrm{o}$ anestro prolongado e $\mathrm{o}$ anestro pós-parto.

Os resultados apontam para a necessidade de melhorias das técnicas de manejo pós-parto e acompanhamento reprodutivo, com constante exame ginecológico nos rebanhos, identificando animais com doenças reprodutivas, retirando-os do plantel, reduzindo desta forma, em médio prazo, o número de casos de enfermidades reprodutivas de caráter hereditário, devido ao descarte das matrizes. Deste modo se contribui com o melhoramento genético do plantel, extremamente importante nos animais de genética superior (P.O.), responsáveis pelo incremento de qualidade dos rebanhos (Grunert et al., 2005).

A adoção de biotecnologias atualmente disponíveis, como a IATF (inseminação artificial em tempo fixo), é importante ferramenta para garantir satisfatórios índices reprodutivos, eliminando a necessidade de observação de estros e permitindo ao final da estação de monta maior taxa de prenhez (Baruselli, et al., 2007).

\section{Conflito de Interesse}

Os autores declaram não existir conflito de interesse.

\section{Comitê de ética}

Todos os animais foram manipulados em uma fazenda comercial durante a rotina de diagnóstico de prenhez e foram obtidos dados a partir de fichas de registro da fazenda. Todos os animais foram tratados com boas práticas de cuidados veterinários e respeitando o bem-estar animal.

\section{Agradecimentos}

A.H.H. Minervino agradece ao $\mathrm{CNPq}$ pela concessão de bolsa de produtividade em pesquisa.

\section{Referências}

Aragón, V.E.F.; Graça, D.S.; Norte, A.L.; Santiago, G.S.; Paula, O.J. Suplementação com cromo e desempenho reprodutivo de vacas zebu primíparas mantidas a pasto. Arquivos Brasileiros de Medicina Veterinária e Zootecnia, 53(5): 624-628, 2001.

Baruselli, P.S.; Gimenes, L.U.; Sales, J.N.S. Fisiologia reprodutiva de fêmeas taurinas e zebuínas. Revista Brasileira de Reprodução Animal, 31(2): 205-211, 2007.

Bustamante, J.R.B.; Fonseca, F.A.; Fontes, C.A.; Espeschit, C.J.; Cardoso, V.C. Effect of body condition at calving and at weaning on the reproductive efficiency of Nelore cows. Revista Brasileira de Zootecnia, 26(6): 1090-1095, 1997.

Cavalcante, F.A.; Martins Filho, R.; Campello, C.C.; Lobo, R.N.B.; Martins, G.A. Intervalo de partos em rebanho nelore na Amazônia oriental. Revista Brasileira de Zootecnia, 29(5): 1327-1331, 2000.

Cavalcante F.A.; Martins Filho, R.; Campello, C.C. Período de gestação em rebanho nelore na Amazônia oriental. Revista Brasileira de Zootecnia, 30(5): 1456-1459, 2001.

Fonseca, J.F.; Alves, C.A.; Paulino, M.F. Nutrition and reproductive efficiency in cows (a review). Veterinária Notícias, 8(1): 125-133, 2002.

Gilbert, R.O.; Shin, S.T.; Guard, C.L. Erb, H.N. Frajblat, M. Prevalence of endometritis and its effects on reproductive performance of dairy cows. Theriogenology, 64(9): 1879-1888, 2005.

Gordon, I. Reproduction in cattle \& buffaloes. Controlled reproduction in farm animals series. Vol. 1. CAB International, Wakkingford, 1996, $492 \mathrm{p}$.

Grunert, E. Zyklus und bruststörungen. In_. E. Grunert \& M. Berchtold. fertilitätsstörungen beim weiblichen Rind. Berlin \& Hamburg: Parey Verlag, 1999, 430 p. 
Grunert, E.; Birgel, E.H.; Vale, W.G. Patologia e clínica da reprodução dos mamíferos domésticos. São Paulo: Varela, 2005. 560p.

Heather, G.; Erb, H.N. Simulated effects on dairy cattle health of extending the voluntary waiting period with recombinant bovine somatotropin. Preventive Veterinary Medicine, 46(1): 29-50, 2000.

IBGE. Instituto Brasileiro de Geografia e Estatística. Produção da Pecuária Municipal, 2016. Disponível em: https://www.ibge.gov.br/estatisticasnovoportal/economicas/agricultura-epecuaria/9107-producao-da-pecuariamunicipal.html. Acesso em: 15 dez. 2017

Laporte, H.M.; Hogeveen. H.; Schukken, Y.H.; Noordhuizen, J.P.T.M. Cystic ovarian disease in Dutch dairy cattle, I. Incidence, risk-factors and consequences. Livestock Production Science, 38(3): 191-197, 1994.

Leite, T.E.; Moraes, J.C.F.; Pimentel, C.A. Eficiência produtiva e reprodutiva em vacas leiteiras. Ciência Rural, 31(3): 467-472, 2001.

Minervino, A.H.H.; Cardoso, E.C.; Ortolani, E.L. Características do sistema produtivo da pecuária no município de Santarém, Pará. Acta Amazônica, 38(1): 11-16, 2008.

Minitab. The Student Edition of Minitab Statistical software adapted for education 13.0 Release. User's Manual. New York: Addison - Wesley, 2000.

Nebel, R. L. A chave para o sucesso em um programa de manejo reprodutivo. Advances In Dairy Technology, 15: 1-11, 2003.

Noronha, C.F.; Nogueira, L.A.G.; Pinho, T.G.; Vieites, F. Effect of uterine manipulation on reproductive efficiency in Nelore cows.
Revista Brasileira de Ciência Veterinária, 6(1): 31-35. 1999.

Oliveira Júnior, R.C.; Correa, J.R.V. Aptidão agrícola dos solos de Município de Belterra, Estado do Pará. Belém: Embrapa Amazônia Oriental, 2001, 21p. (Embrapa Amazônia Oriental. Documentos, 91).

Palhano, H.B. Epidemiologia. In: Palhano, H.B. Reprodução em bovinos. $2^{a}$ ed. Rio de Janeiro: LF. Livros de veterinária, 2008.

Rhodes, F.M.; Entwistle, K.W.; Kinder, J.E. Changes in ovarian function and gonadotropin secretion preceding the onset of nutritionally induced anoestrus in Bos indicus heifers. Biology Reproduction, 55: 1437-1443, 1996.

Sartori, R.; Guardieiro, M.M. Fatores nutricionais associados à reprodução da fêmea bovina. Revista Brasileira de Zootecnia, 39: 422432, 2010.

Sheldon, M.; Wathes, D.C.; Dobson, H. The management of bovine reproduction in elite herds. Veterinary Journal, 171(1): 70-78, 2006.

Vale, W.G. Prevalência de "repeat breeders" em rebanhos nelore na região amazônica. Revista Brasileira de Reprodução Animal, 3(4): 2327, 1979.

Vale, W.G., Ohashi, O.M., Ribeiro, H.F.R.; Sousa, J.S. Causes and incidence of infertility and subfertility in zebu crossed cows in Amazon region of Brazil. Veterinary Medicine Review, 2: 133-143, 1984.

Wiltbank, M.C.; Gumen, A.; Sartori, R. Physiological classification of anovulatory conditions in cattle. Theriogenology, 57: 2152,2002 